\title{
The QCD pomeron in ultraperipheral heavy ion collisions: IV. Photonuclear production of vector mesons
}

\author{
V.P. Gonçalves ${ }^{1}$, M.V.T. Machado 2,3, a \\ 1 Instituto de Física e Matemática, Universidade Federal de Pelotas, Caixa Postal 354, CEP 96010-090, Pelotas, RS, Brazil \\ 2 Universidade Estadual do Rio Grande do Sul - UERGS, Unidade de Bento Gonçalves, CEP 95700-000, Bento Gonçalves, RS, \\ Brazil \\ 3 High Energy Physics Phenomenology Group, GFPAE IF-UFRGS, Caixa Postal 15051, CEP 91501-970, Porto Alegre, RS, \\ Brazil
}

Received: 12 January 2005 / Revised version: 2 February 2005 /

Published online: 9 March 2005 - (C) Springer-Verlag / Società Italiana di Fisica 2005

\begin{abstract}
The photonuclear production of vector mesons in ultraperipheral heavy-ion collisions is investigated within the QCD color dipole picture, with particular emphasis on the saturation model. The integrated cross section and the rapidity distribution for the $A A \rightarrow V A A(V=\rho, \omega, \phi, J / \Psi)$ process are computed and theoretical estimates for scattering on both light and heavy nuclei are given for the energies of RHIC and LHC. A comparison with the recent STAR data on coherent production of $\rho$ mesons is also presented. Furthermore, we calculate the photoproduction of vector mesons in proton-proton collisions at RHIC, Tevatron and LHC energies.
\end{abstract}

\section{Introduction}

In ultraperipheral relativistic heavy-ion collisions (UPC's) the ions do not interact directly with each other and move essentially undisturbed along the beam direction. The only possible interaction is due to the long range electromagnetic interaction and diffractive processes (for a review see, e.g. [1]). Due to the coherent action of all the protons in the nucleus, the electromagnetic field is very strong and the resulting flux of equivalent photons is large. A photon stemming from the electromagnetic field of one of the two colliding nuclei can penetrate into the other nucleus and interact with one or more of its hadrons, giving rise to photon-nucleus collisions to an energy region hitherto unexplored experimentally. For example, the interaction of quasi-real photons with protons has been studied extensively at the electron-proton collider at HERA, with $\sqrt{s}=300 \mathrm{GeV}$. The obtained $\gamma p$ center of mass energies extends up to $W_{\gamma p} \approx 200 \mathrm{GeV}$, an order of magnitude larger than those reached by fixed target experiments. Due to the larger number of photons coming from one of the colliding nuclei in heavy-ion collisions, a similar and more detailed study will be possible in these collisions, with $W_{\gamma N}$ reaching $950 \mathrm{GeV}$ for the Large Hadron Collider (LHC) operating in its heavy-ion mode. Similarly, the strong electromagnetic fields generated by high-energy protons allow us to study photon-nucleon processes in proton-proton interactions in a large kinematical range. These events can be experimentally studied by selecting

\footnotetext{
a e-mail: magno-machado@uergs.edu.br
}

events with low multiplicity and small total transverse momentum.

Over the past few years a comprehensive analysis of the heavy quark [2-6] and vector meson [3,7-11] production in ultraperipheral heavy-ion collisions was made considering different theoretical approaches. In particular, much effort has been devoted to obtain signatures of the QCD pomeron in such processes $[5,6,9]$, which can be used to constrain the QCD dynamics at high energies. Understanding the behavior of high-energy hadron reactions from a fundamental perspective within QCD is an important goal of particle physics. In the late 1970s, Lipatov and collaborators [12] established the papers which form the core of our knowledge of the Regge limit (high-energy limit) of QCD. The physical effect that they describe is often referred to as the QCD pomeron, with the evolution described by the BFKL equation. Since this equation predicts that for $s \rightarrow \infty$ the corresponding cross section rises with a power law of the energy, violating the Froissart bound, new dynamical effects associated with the unitarity corrections are expected to stop further growth of the cross sections [13]. This expectation can be easily understood: while for large transverse momentum $k_{\perp}$, the BFKL equation predicts that the mechanism $g \rightarrow g g$ populates the transverse space with a large number of small size gluons per unit of rapidity (the transverse size of a gluon with momentum $k_{\perp}$ is proportional to $1 / k_{\perp}^{2}$ ), for small $k_{\perp}$ the produced gluons overlap and fusion processes, $g g \rightarrow g$, are equally important. Considering this process, the rise of the gluon distribution with transverse momenta below 
a typical scale, energy dependent, called the saturation scale $Q_{\text {sat }}$ is reduced, restoring the unitarity. It is important to emphasize that Golec-Biernat and Wusthoff [14] have shown that a saturation model is able to describe the DESY ep collider HERA data, in particular the transition from the perturbative to the non-perturbative photoproduction region (for improvements of this model, see $[15,16])$. Moreover, its parameter-free application to diffractive DIS has been also quite successful [14] as well as its extension to virtual Compton scattering [17], vector meson production [18-20], charm and the longitudinal structure function $[21,22]$ and two-photon collisions [23]. However, once other approaches containing very distinct assumptions also describe the same set of HERA data, the description of the QCD pomeron still is an open question and it deserves more detailed analyses. An alternative way to constrain the QCD dynamics are studies on electron-nucleus interactions, since the saturation effects are amplified in a nuclear medium; $Q_{s A}^{2} \propto A^{1 / 3}$. Several works have been made along these lines, studying the behavior of the observables in the kinematic region of the planned $e A$ colliders at HERA and RHIC (for recent reviews see, e.g., $[24,25])$. In particular, recently we have analyzed the nuclear exclusive vector meson production considering two distinct theoretical scenarios [26]. Our results have demonstrated that the experimental analyses of nuclear exclusive vector meson photoproduction in the future electron-nucleus colliders eRHIC and HERA-A could be useful to discriminate between the different theoretical scenarios, mainly if heavy nuclei are considered. It strongly motivates the extension of these studies for ultraperipheral heavy-ion collisions which can be analyzed in the current and/or scheduled colliders.

Recently, the STAR Collaboration released the first data on the cross section of the coherent $\rho$ production in gold-gold ultraperipheral collisions at $\sqrt{s}=130 \mathrm{GeV}$ [27], providing the first opportunity to check the basic features and main approximations of the distinct approaches describing nuclear vector meson photoproduction. The main aspect is that real photons have a complicated nature. In a first approximation, the photon is a point-like particle, although in field theory it may fluctuate also into a fermion pair (see discussions in $[1,28]$ ). In the case where there is a photon transition in a colorless antiquark-quark pair, the propagation of this colorless hadronic wave packet in a nuclear medium can be treated either in the hadronic basis as a result of Gribov's inelastic corrections or in QCD in terms of the partonic basis, which are complementary. Let us briefly discuss these two representations (for a detailed discussion, see [29]). The time scale characterizing the evolution of a $q \bar{q}$ wave packet can be estimated based on the uncertainty principle and Lorentz dilation. The lifetime of the photon fluctuation is given by $t_{\mathrm{c}}=\nu /\left(Q^{2}+m_{V}^{2}\right)$, where $\nu$ is the photon energy, $m_{V}$ is the mass of the fluctuation and $Q^{2}$ is the photon virtuality. It is usually called the coherence time. Using light-cone kinematics we can define the coherence length, which is given by $l_{\mathrm{c}}=t_{\mathrm{c}}$. Moreover, one cannot decide whether a ground state $V$ is produced or the next-excited state $V^{\prime}$, unless the process lasts longer than the inverse mass difference between these states. In the rest frame of the nucleus, this formation time is Lorentz dilated and is given by $t_{\mathrm{f}}=2 \nu /\left(m_{V^{\prime}}^{2}-m_{V}^{2}\right)$. Similarly, we can define a formation length given by $l_{\mathrm{f}}=t_{\mathrm{f}}$. In the hadronic basis, the same process looks quite different. The incident photon may produce different states on a bound nucleon, the $V$ meson ground state or an excited state. Those states propagate through the nucleus experiencing multiple-diagonal and off-diagonal diffractive interactions, and eventually the ground state is detected. According to the quark-hadron duality, we expect these two descriptions to be equivalent. However, as these two approaches have been used assuming different approximations, their comparison may provide a scale for the theoretical uncertainty involved. Furthermore, it is important to emphasize that at high photon energy $\nu$, both $l_{\mathrm{c}}$ and $l_{\mathrm{f}}$ greatly exceed the nuclear radius $R_{A}$, which implies in the partonic basis that the transverse size of the $q \bar{q}$ pair does not change during the interaction with the target. This enables one to introduce the QCD dipole picture [30], where the process is factorized into the photon fluctuation in a $q \bar{q}$ pair and the dipole cross section. These aspects become in the interpretation in the partonic basis more intuitive and straightforward than in the hadronic basis. Since the photoproduction of vector mesons in ultraperipheral heavy-ion collisions have been analyzed in the literature using only the hadronic basis, it motivates the study of this process using the partonic one.

Another important point which motivates our analysis is that in the current studies of vector meson production in UPC's the extrapolations of the predictions for LHC energies in general assume a power-like behavior for the $\gamma p(A) \rightarrow V p(A)$ cross sections. Despite the good agreement for the currently available energies, an extrapolation to higher energies of the experimental fits implies a large growth for the cross section which would violate the unitarity at sufficiently high energies. Therefore, dynamical modifications associated to the unitarity corrections are also expected to be present in vector meson production [18-20]. As discussed before, these effects should be enhanced in nuclear processes $[13,31,26]$.

In this paper the photonuclear production of vector mesons in UPC's is investigated within the QCD color dipole picture [30], with particular emphasis on the saturation model [14]. The exclusive vector meson production by real and virtual photons is an outstanding process providing important information on the transition region from the soft dynamics (at low virtualities of the photon $\left.Q^{2}\right)$ to the hard perturbative regime at high $Q^{2}[32,33]$ (for a recent review, see [34]). In principle, a perturbative approach is only justified if a hard scale is present in the process, e.g. the photon virtuality and/or a large mass of the vector meson. For photoproduction of light mesons, such a scale is not present, and one has to rely on nonperturbative models. In some pQCD approaches, as the saturation model, even this soft process can be described, where the transition is set by the saturation scale. In the color dipole approach, the degrees of freedom are the pho- 
ton (color dipole) and meson wavefunctions as well as the dipole-nuclei cross section. Such an approach enables one to include nuclear effects and the parton saturation phenomenon. The latter one is characterized by the typical momentum (saturation) scale $Q_{\text {sat }}$, which has been constrained by experimental results in deep inelastic scattering (DIS) and diffractive DIS [14]. Here, we will use an extension of the phenomenological saturation model for nuclear targets [35]. This model reasonably describes the experimental data for the nuclear structure function and has been used to predict the nuclear inclusive and diffractive cross sections for heavy quark photoproduction [36]. The nuclear saturation scale, $Q_{\mathrm{s} A}$, provides the transition between the color transparency and the saturation regimes in the nuclear scattering. Concerning vector meson production, our starting point are the recent works in $[20,26]$, where this approach was applied for the proton and nuclear case. It is worth mentioning that although light meson photoproduction is a soft process by definition, it is consistently described in the QCD color dipole picture whether or not there is a suitable model for the soft-hard transition, as occurring in the saturation model. As a by-product, we extend our analysis for the photoproduction of vector mesons in proton-proton collisions at RHIC, Tevatron and LHC energies.

This paper is organized as follows. In the next section, we present a brief review of the ultraperipheral heavy-ion collisions and the main formulae to describe the photonhadron process in these reactions. Moreover, the photon spectrum in proton-proton collisions is discussed. In Sect. 3 we discuss the photoproduction of vector mesons in the QCD color dipole picture and the saturation model is shortly reviewed. In Sect. 4 we present our results for the integrated cross section and the rapidity distribution for the $A A \rightarrow A A V$ and $p p \rightarrow p p V$ processes, where $V=\rho, \omega, \phi, J / \Psi$. Moreover, a comparison is presented of our predictions with the STAR data for coherent $\rho$ photoproduction on nuclei at energy $\sqrt{s_{N N}}=130 \mathrm{GeV}$ and a discussion concerning related approaches used in its description is addressed. Finally, in the last section we present a summary of the main results and conclusions.

\section{Photonuclear vector meson production at UPC's}

In heavy-ion collisions the large number of photons coming from one of the colliding nuclei will allow us to study photoproduction, with energies $W_{\gamma N}$ reaching to $950 \mathrm{GeV}$ for the LHC. The photonuclear cross sections are given by the convolution between the photon flux from one of the nuclei and the cross section for photon-nuclei scattering. The final expression for the production of vector mesons in ultraperipheral heavy-ion collisions is then given by

$$
\begin{aligned}
& \sigma_{A A \rightarrow A A V}\left(\sqrt{S_{N N}}\right) \\
& =\int_{\omega_{\min }}^{\infty} \mathrm{d} \omega \frac{\mathrm{d} N(\omega)}{\mathrm{d} \omega} \sigma_{\gamma A \rightarrow V A}\left(W_{\gamma A}^{2}=2 \omega \sqrt{S_{N N}}\right),
\end{aligned}
$$

where $\omega$ is the photon energy with $\omega_{\min }=m_{V}^{2} / 4 \gamma_{\mathrm{L}} m_{p}$ and $\sqrt{S_{N N}}$ is the ion-ion CMS energy. The Lorentz factor for LHC is $\gamma_{\mathrm{L}}=2930$, giving the maximum CMS $\gamma N$ energy $W_{\gamma A} \lesssim 950 \mathrm{GeV}$. In this process we have that the nuclei are not disrupted and the final state consists solely of the two nuclei and the vector meson decay products. Consequently, we have that the final state is characterized by a small number of centrally produced particles, with rapidity gaps separating the central final state from both beams. Moreover, due to the coherence requirement, the transverse momentum is limited to values smaller than $p_{\mathrm{T}}=\sqrt{2} / R_{A}$, where $R_{A}$ is the nuclear radius. Therefore, these reactions can be studied experimentally by selecting events with low multiplicity and small total $p_{\mathrm{T}}$.

The photon flux is given by the Weizsäcker-Williams method [1]. The flux from a charge $Z$ nucleus a distance $b$ away is

$$
\frac{\mathrm{d}^{3} N\left(\omega, b^{2}\right)}{\mathrm{d} \omega \mathrm{d}^{2} b}=\frac{Z^{2} \alpha_{\mathrm{em}} \eta^{2}}{\pi^{2} \omega b^{2}}\left[K_{1}^{2}(\eta)+\frac{1}{\gamma_{\mathrm{L}}^{2}} K_{0}^{2}(\eta)\right]
$$

where $\gamma_{\mathrm{L}}$ is the Lorentz boost of a single beam and $\eta=\omega b / \gamma_{\mathrm{L}} ; K_{0,1}(\eta)$ are the modified Bessel functions. The requirement that photoproduction is not accompanied by hadronic interaction (ultraperipheral collision) can be done by restricting the impact parameter $b$ to be larger than twice the nuclear radius, $R_{A}=1.2 A^{1 / 3} \mathrm{fm}$. Therefore, the total photon flux interacting with the target nucleus is given by (2) integrated over the transverse area of the target for all impact parameters subject to the constraint that the two nuclei do not interact hadronically. An analytic approximation for $A A$ collisions can be obtained using as integration limit $b>2 R_{A}$, producing

$$
\begin{aligned}
& \frac{\mathrm{d} N(\omega)}{\mathrm{d} \omega}=\frac{2 Z^{2} \alpha_{\mathrm{em}}}{\pi \omega} \\
& \times\left[\bar{\eta} K_{0}(\bar{\eta}) K_{1}(\bar{\eta})+\frac{\bar{\eta}^{2}}{2}\left(K_{1}^{2}(\bar{\eta})-K_{0}^{2}(\bar{\eta})\right)\right],
\end{aligned}
$$

where $\bar{\eta}=2 \omega R_{A} / \gamma_{\mathrm{L}}$. It is worth mentioning that the difference between the complete numeric and the analytical calculation presented above for the photon flux is less than $15 \%$ for most of the purposes [1].

In a similar way, vector meson production also occur when considering energetic protons in $p p(\bar{p})$ colliders [11]. In this case the photon spectrum is given by [37]

$$
\begin{aligned}
& \frac{\mathrm{d} N(\omega)}{\mathrm{d} \omega}=\frac{\alpha_{\mathrm{em}}}{2 \pi \omega}\left[1+\left(1-\frac{2 \omega}{\sqrt{S_{N N}}}\right)^{2}\right] \\
& \times\left(\ln \Omega-\frac{11}{6}+\frac{3}{\Omega}-\frac{3}{2 \Omega^{2}}+\frac{1}{3 \Omega^{3}}\right),
\end{aligned}
$$

with the notation $\Omega=1+\left[\left(0.71 \mathrm{GeV}^{2}\right) / Q_{\min }^{2}\right]$ and $Q_{\min }^{2}=\omega^{2} /\left[\gamma_{\mathrm{L}}^{2}\left(1-2 \omega / \sqrt{S_{N N}}\right)\right] \approx\left(\omega / \gamma_{\mathrm{L}}\right)^{2}$. The expression above is derived considering the Weizsäcker-Williams method of virtual photons and using an elastic proton form factor (for more details, see $[11,37]$ ). It is important to emphasize that the expression (4) is based on a heuristic approximation, which leads to an overestimation of the 
cross section at high energies $(\approx 11 \%$ at $\sqrt{s}=1.3 \mathrm{TeV})$ in comparison with the more rigorous derivation of the photon spectrum for elastic scattering on protons derived in [38]. For a more detailed comparison among the different photon spectra, see [39].

\section{Vector meson production in the color dipole approach}

Let us consider the scattering process $\gamma p \rightarrow V p$ in the QCD dipole approach, where $V$ stands for both light and heavy mesons. The scattering process can be seen in the target rest frame as a succession in time of three factorizable subprocesses:

(i) the photon fluctuates in a quark-antiquark pair (the dipole),

(ii) this color dipole interacts with the target and

(iii) the pair converts into vector meson final state. Using as kinematic variables the $\gamma^{*} N$ CMS energy squared $s=$ $W_{\gamma N}^{2}=(p+q)^{2}$, where $p$ and $q$ are the target and the photon momenta, respectively, the photon virtuality squared $Q^{2}=-q^{2}$ and the Bjorken variable $x=Q^{2} /\left(W_{\gamma N}^{2}+Q^{2}\right)$, the corresponding imaginary part of the amplitude at zero momentum transfer reads [30]

$$
\begin{aligned}
& \operatorname{Im} \mathcal{A}(\gamma p \rightarrow V p) \\
& =\sum_{h, \bar{h}} \int \mathrm{d} z \mathrm{~d}^{2} \boldsymbol{r} \Psi_{h, \bar{h}}^{\gamma}\left(z, \boldsymbol{r}, Q^{2}\right) \sigma_{\operatorname{dip}}^{\operatorname{target}}(\tilde{x}, \boldsymbol{r}) \Psi_{h, \bar{h}}^{V *}(z, \boldsymbol{r}),
\end{aligned}
$$

where $\Psi_{h, \bar{h}}^{\gamma}(z, \boldsymbol{r})$ and $\Psi_{h, \bar{h}}^{V}(z, \boldsymbol{r})$ are the light-cone wavefunctions of the photon and vector meson, respectively. The quark and antiquark helicities are labeled by $h$ and $\bar{h}$ and reference to the meson and photon helicities is implicitly understood. The variable $\boldsymbol{r}$ defines the relative transverse separation of the pair (dipole) and $z(1-z)$ is the longitudinal momentum fractions of the quark (antiquark). The basic blocks are the photon wavefunction, $\Psi^{\gamma}$, the meson wavefunction, $\Psi_{\mathrm{T}, \mathrm{L}}^{V}$, and the dipole-target cross section, $\sigma_{\text {dip }}^{\text {target }}$.

In the dipole formalism, the light-cone wavefunctions $\Psi_{h, \bar{h}}(z, \boldsymbol{r})$ in the mixed representation $(z, \boldsymbol{r})$ are obtained through two dimensional Fourier transform of the momentum space light-cone wavefunctions, $\Psi_{h, \bar{h}}(z, \boldsymbol{k})$ which can be completely determined using light-cone perturbation theory (see more details in, e.g., $[19,20,32]$ ). On the other hand, for vector mesons, the light-cone wavefunctions are not known in a systematic way and they are thus obtained through models. Here, we follow the analytically simple DGKP approach [40], which is found to describe in good agreement vector meson production as pointed out in [20]. In this particular approach, one assumes that the dependencies on $\boldsymbol{r}$ and $z$ of the wavefunction are factorized, with a Gaussian dependence on $\boldsymbol{r}$ (for a detailed discussion see $[20,26])$.

Finally, the imaginary part of the forward amplitude can be obtained by putting the expressions for photon and vector meson (DGKP) wavefunctions into (5). Moreover, summation over the quark/antiquark helicities and an average over the transverse polarization states of the photon should be taken into account. The transverse component (the longitudinal one does not contribute for photoproduction) is then written as $[20,26]$

$$
\begin{aligned}
& \operatorname{Im} \mathcal{A}_{\mathrm{T}}(s, t=0) \\
& =\int \mathrm{d}^{2} \boldsymbol{r} \int_{0}^{1} \mathrm{~d} z \alpha_{\mathrm{em}}^{1 / 2} f_{V} f_{\mathrm{T}}(z) \exp \left[\frac{-\omega_{\mathrm{T}}^{2} \boldsymbol{r}^{2}}{2}\right] \\
& \times\left\{\frac{\omega_{\mathrm{T}}^{2} \varepsilon r}{m_{V}}\left[z^{2}+(1-z)^{2}\right] K_{1}(\varepsilon r)+\frac{m_{f}^{2}}{m_{V}} K_{0}(\varepsilon r)\right\} \\
& \quad \times \sigma_{\text {dip }}^{\text {target }}(\tilde{x}, \boldsymbol{r}),
\end{aligned}
$$

with $\sigma_{\text {dip }}^{\text {target }}$ being the dipole-proton cross section in the nucleon case and the dipole-nucleus cross section for scattering on nuclei. In the photoproduction case, $\varepsilon=m_{f}$, where $m_{f}$ is the quark mass of flavor $f$. The corresponding parameters for the vector mesons wavefunctions $\left(m_{V}\right.$, $\omega_{\mathrm{T}}, f_{V}$, etc) are presented in Table 1 of [26].

In order to obtain the total cross section, we assume an exponential parameterization for the small $|t|$ behavior of the amplitude. After integration over $|t|$, the total cross section for vector meson production by real/virtual photons in the nucleon (proton) case reads

$$
\sigma(\gamma p \rightarrow V p)=\frac{[\operatorname{Im} \mathcal{A}(s, t=0)]^{2}}{16 \pi B_{V}}\left(1+\beta^{2}\right),
$$

where $\beta$ is the ratio of real to imaginary part of the amplitude and $B_{V}$ labels the slope parameter. The values considered for the slope parameter are taken from the parameterization used in [18]. For the $\rho$ case, we have taken a different value in order to describe simultaneously $\mathrm{H} 1$ and ZEUS photoproduction data.

In addition, (6) represents only the leading imaginary part of the positive-signature amplitude, and its real part can be restored using dispersion relations $\operatorname{Re} \mathcal{A}=$ $\tan (\pi \lambda / 2) \operatorname{Im} \mathcal{A}$. Thus, for the $\beta$ parameter we have used the simple ansatz

$$
\beta=\tan \left(\frac{\pi \lambda_{\mathrm{eff}}}{2}\right)
$$

where

$$
\lambda_{\text {eff }}=\frac{\partial \ln [\operatorname{Im} \mathcal{A}(s, t=0)]}{\partial \ln s},
$$

with $\lambda_{\text {eff }}=\lambda_{\text {eff }}\left(W_{\gamma N}, Q^{2}\right)$ the effective power of the imaginary amplitude, which depends on both energy and photon virtuality. The correction coming from real part in photoproduction, where only transverse component contributes, is about $3 \%$ for light mesons and it reaches $13 \%$ for $J / \Psi$ at high energies. It is worth mentioning that a different computation of the $\beta$ parameter, as in [20], produces a larger effect even in the photoproduction case. An additional correction is still required for heavy mesons, like $J / \Psi$. Namely, skewedness effects which takes into account the off-forward features of the process (different transverse 
momenta of the exchanged gluons in the $t$-channel), are increasingly important in this case. Here, we follow the studies in [41], where the ratio of off-forward to forward gluon distributions reads [41]

$$
R_{\mathrm{g}}\left(\lambda_{\mathrm{eff}}\right)=\frac{2^{2 \lambda_{\mathrm{eff}}+3}}{\sqrt{\pi}} \frac{\Gamma\left(\lambda_{\mathrm{eff}}+\frac{5}{2}\right)}{\Gamma\left(\lambda_{\mathrm{eff}}+4\right)},
$$

and we will multiply the total cross section by the factor $R_{g}^{2}$ for the heavy meson case.

In the case of nuclear targets, $B_{V}$ is dominated by the nuclear size, with $B \sim R_{A}^{2}$ and the non-forward differential cross section is dominated by the nuclear form factor, which is the Fourier transform of the nuclear density profile. Here we use the analytical approximation of the Woods-Saxon distribution as a hard sphere, with radius $R_{A}$, convoluted with a Yukawa potential with range $a=0.7 \mathrm{fm}$. Thus, the nuclear form factor reads [7]

$$
\begin{aligned}
& F(q=\sqrt{|t|}) \\
& =\frac{4 \pi \rho_{0}}{A q^{3}}\left[\sin \left(q R_{A}\right)-q R_{A} \cos \left(q R_{A}\right)\right]\left[\frac{1}{1+a^{2} q^{2}}\right],
\end{aligned}
$$

where $\rho_{0}=0.16 \mathrm{fm}^{-3}$. Consequently, the photonuclear cross section is given by

$$
\begin{aligned}
& \sigma(\gamma A \rightarrow V A) \\
& =\frac{\left[\operatorname{Im} \mathcal{A}_{\text {nuc }}(s, t=0)\right]^{2}}{16 \pi}\left(1+\beta^{2}\right) \int_{t_{\min }}^{\infty} \mathrm{d} t|F(t)|^{2},
\end{aligned}
$$

with $t_{\min }=\left(m_{V}^{2} / 2 \omega\right)^{2}$, where $\omega$ is the photon energy.

Having introduced the main expressions for computing vector meson production in the color dipole approach, in what follows we present a brief review of the saturation model and its extension for the scattering on nuclei targets. In the present work, we follow the quite successful saturation model [14], which interpolates between the small and large dipole configurations, providing color transparency behavior, $\sigma_{\text {dip }} \sim \boldsymbol{r}^{2}$, as $\boldsymbol{r} \rightarrow 0$ and constant behavior, $\sigma_{\text {dip }} \sim \sigma_{0}$, at large dipole separations. The parameters of the model have been obtained from an adjustment to small $x$ HERA data. The parameterization for the dipole cross section takes the eikonal-like form [14],

$$
\begin{aligned}
\sigma_{\text {dip }}^{\text {proton }}\left(\tilde{x}, \boldsymbol{r}^{2}\right) & =\sigma_{0}\left[1-\exp \left(-\frac{Q_{\text {sat }}^{2}(\tilde{x}) \boldsymbol{r}^{2}}{4}\right)\right], \\
Q_{\text {sat }}^{2}(\tilde{x}) & =\left(\frac{x_{0}}{\tilde{x}}\right)^{\lambda} \mathrm{GeV}^{2},
\end{aligned}
$$

where the saturation scale $Q_{\text {sat }}^{2}$ defines the onset of the saturation phenomenon, which depends on energy. The parameters, obtained from a fit to the small- $x$ HERA data, are $\sigma_{0}=23.03(29.12) \mathrm{mb}, \lambda=0.288(0.277)$ and $x_{0}=$ $3.04 \cdot 10^{-4}\left(0.41 \cdot 10^{-4}\right)$ for a 3 -flavor (4-flavor) analysis. An additional parameter is the effective light quark mass, $m_{\mathrm{f}}=0.14 \mathrm{GeV}$, which plays the role of a regulator for the photoproduction $\left(Q^{2}=0\right)$ cross section. The charm quark mass is considered to be $m_{c}=1.5 \mathrm{GeV}$. A smooth transition to the photoproduction limit is obtained via the scaling variable $[14] \tilde{x}=\left[\left(Q^{2}+4 m_{f}^{2}\right) /\left(Q^{2}+W_{\gamma N}^{2}\right)\right]$.
The saturation model is suitable in the region below $x=0.01$ and the large $x$ limit needs still a consistent treatment. Making use of the dimensional-cutting rules, here we supplement the dipole cross section, (12), with a threshold factor $(1-x)^{n_{\text {thres }}}$, taking $n_{\text {thres }}=5$ for a 3 -flavor analysis and $n_{\text {thres }}=7$ for a 4 -flavor one. This procedure ensures a consistent description of heavy quark production at the fixed target data [42].

Let us discuss the extension of the saturation model for the photon-nucleus interactions. Here, we follow the simple procedure proposed in [35], which consists of an extension to nuclei of the saturation model discussed above, using the Glauber-Gribov picture [43], without any new parameter. In this approach, the nuclear version is obtained replacing the dipole-nucleon cross section in (5) by the nuclear one,

$$
\begin{aligned}
& \sigma_{\text {dip }}^{\text {nucleus }}\left(\tilde{x}, \boldsymbol{r}^{2} ; A\right) \\
& =2 \int \mathrm{d}^{2} b\left\{1-\exp \left[-\frac{1}{2} T_{A}(b) \sigma_{\text {dip }}^{\text {proton }}\left(\tilde{x}, \boldsymbol{r}^{2}\right)\right]\right\},
\end{aligned}
$$

where $b$ is the impact parameter of the center of the dipole relative to the center of the nucleus and the integrand gives the total dipole-nucleus cross section for a fixed impact parameter. The nuclear profile function is labeled by $T_{A}(b)$, which will be obtained from a 3-parameter Fermi distribution for the nuclear density [44]. The above equation sums up all the multiple elastic rescattering diagrams of the $q \bar{q}$ pair and is justified for a large coherence length, where the transverse separation $\boldsymbol{r}$ of partons in the multiparton Fock state of the photon becomes as good a conserved quantity as the angular momentum, i.e. the size of the pair $\boldsymbol{r}$ becomes an eigenvalue of the scattering matrix. It is important to emphasize that for very small values of $x$, other diagrams beyond the multiple pomeron exchange considered here should contribute (e.g. pomeron loops) and a more general approach for the high density (saturation) regime must be considered. However, we believe that this approach allows us to obtain lower limits of the high density effects. Therefore, the region of applicability of this model should be at small values of $x$, i.e. a large coherence length, and for not too high values of virtualities, where the implementation of the DGLAP evolution should be required. Consequently, the approach is quite suitable for the analysis of exclusive vector meson photoproduction in the kinematical range of the planned lepton-nucleus colliders (eRHIC and HERA-A) as well as UPC's at RHIC and LHC colliders. It is noticeable that the energy dependence of the cross sections is strongly connected with the saturation scale $Q_{\mathrm{s} A}\left(W_{\gamma N}\right)$. Namely, the saturation effects are larger whether the momentum scale is of order or larger than the corresponding size of the vector meson and the energy growth of the cross section is then slowed down.

\section{Results and discussions}

In this section we present the numerical calculation of the rapidity distribution and total cross section for the photo- 
RHIC: AuAu $\rightarrow$ AuAu + $\left(\mathbf{V}_{\mathrm{M}}=\rho, \omega, \phi, \mathrm{J} / \Psi\right)$
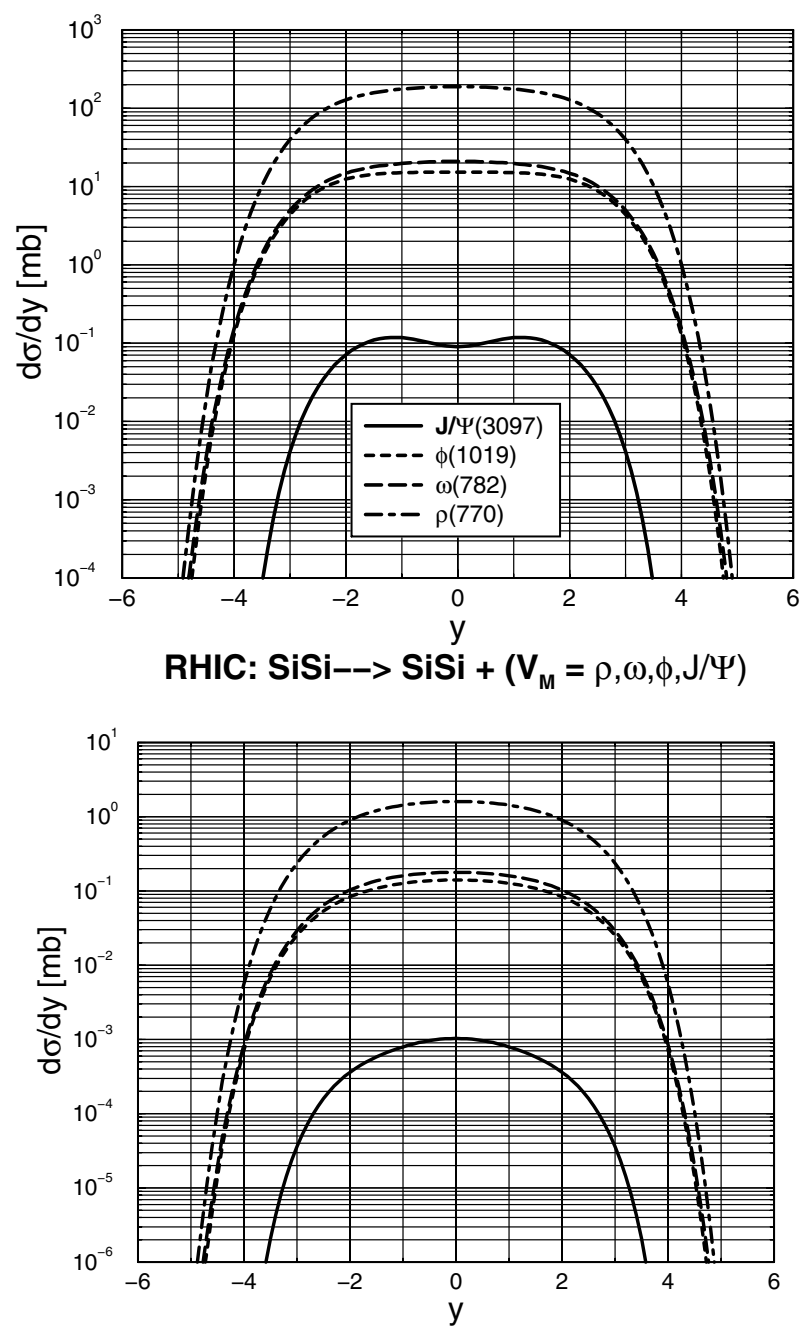

Fig. 1. The rapidity distribution for nuclear vector meson photoproduction on UPC's in $A A$ reactions at RHIC energy $\left(\sqrt{S_{N N}}=0.2 \mathrm{TeV}\right)$

production of vector mesons in ultraperipheral heavy-ion and proton-proton collisions. Our main goal is to obtain estimates from the QCD saturation model for vector meson photoproduction in the kinematical range of the colliders RHIC, LHC and Tevatron. Furthermore, a discussion concerning the comparison of the present results with the currently available models $[7,8]$ based on vector meson dominance (VDM) and parton-hadron duality is also presented.

Initially, let us consider the nuclear photoproduction of vector mesons in ultraperipheral heavy-ion collisions at $\operatorname{RHIC}(\sqrt{s}=200 \mathrm{GeV})$ and LHC $(\sqrt{s}=5500 \mathrm{GeV})$ energies for different nuclei. In Figs. 1 and 2 one presents the predictions for meson rapidity distributions and in Table 1 one shows the corresponding integrated cross section. For LHC the considered nuclei are lead $(\mathrm{Pb})$ and calcium $(\mathrm{Ca})$, whereas for RHIC one takes gold $(\mathrm{Au})$ and silicon $(\mathrm{Si})$.

\section{LHC: $\mathbf{P b P b} \rightarrow \mathbf{P b P b}+\left(\mathbf{V}_{\mathrm{M}}=\rho, \omega, \phi, \mathrm{J} / \Psi\right)$}
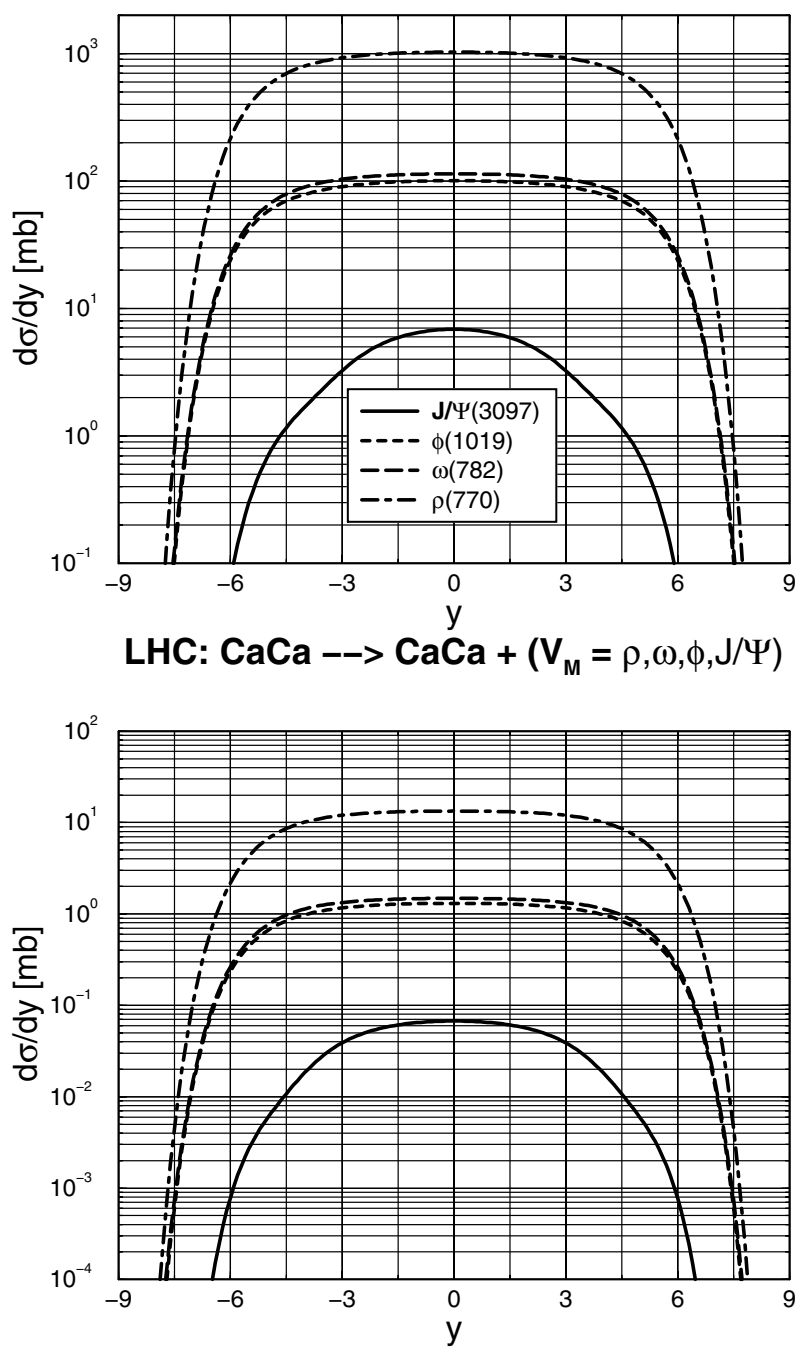

Fig. 2. The rapidity distribution for vector meson photoproduction on $A A$ reactions at $\mathrm{LHC}$ energy $\sqrt{S_{N N}}=5.5 \mathrm{TeV}$

The distributions for light and heavy vector mesons are placed in the same plot for the sake of comparison among their orders of magnitude. The final state rapidity is determined by the simple relation $y=\frac{1}{2} \ln \frac{\omega}{\sqrt{\left|t_{\min }\right|}}=\ln \frac{2 \omega}{m_{V}}$, that is the ratio between the photon energy $\omega$ and the longitudinal energy transfer in the laboratory frame. Consequently, the rapidity distribution reads

$$
\frac{\mathrm{d} \sigma(A A \rightarrow A A V)}{\mathrm{d} y}=\omega \frac{\mathrm{d} \sigma(A A \rightarrow A A V)}{\mathrm{d} \omega},
$$

where $\frac{\mathrm{d} \sigma}{\mathrm{d} \omega}$ is calculated using (1). Interchanging the photon emitter and target corresponds to a reflection around $y=$ 0 , with the total cross section being the sum of these two possibilities.

Let us discuss the numerical results. The rapidity distribution is characterized by a plateau at mid-rapidity at 
both RHIC and LHC, even for the light mesons as the $\rho$ production. Such a plateau is more pronounced at LHC. This is in contrast with the theoretical predictions of $[7$, 8], where due to the matching between the photon spectrum and the photonuclear cross section at lower (RHIC) energies a clear double-peak structure appears. For LHC, that feature disappears since lower photon energies contribute in a smaller amount to the total distribution. This behavior is not present in our results mostly due to the inclusion of the threshold factor in the dipole-nucleus cross section, which enforces the correct behavior at energies near threshold region. This factor also prevents the sharp cut-off at the fragmentation (forward rapidities) region, which has appeared in recent calculations considering the $J / \Psi$ production [11].

The integrated cross sections can be contrasted with the theoretical estimations using GVDM plus GlauberGribov approach of [8] as well as the estimation of [7], which considers VDM plus a classical mechanical calculation for nuclear scattering and uses as input for the $\gamma p \rightarrow V p$ reaction an extrapolation of the experimental DESY-HERA fits for meson photoproduction. Initially let us consider the latter approach (see Table III in [7]). At RHIC energy and Si nucleus, our results are about $20 \%$ lower for $\rho$ and $\omega$, whereas gives a larger $\phi$ cross section and almost the same $J / \Psi$ cross section. However, the situation changes for a gold nucleus, where the present results are about 50\% larger than the estimates in [7]. At LHC energy and for Ca nucleus, our results give a higher cross section by a factor of order $10 \%$, whereas for a lead nucleus the factor reaches a factor 2 for light mesons and almost similarly for the $J / \Psi$ meson. Basically, the values are quite similar for light nuclei. For the heaviest nuclei, the results overestimate those in [7] when one considers light mesons and become similar for the $J / \Psi$ case. These features can be understood through the theoretical procedure when considering the nuclear scattering.

Despite the QCD color dipole model being a different approach than that used in [7], we can qualitatively understand the discrepancy on the results by looking at the $\gamma A \rightarrow V A$ cross section in both models, which is the input for the UPC's calculations in (1). For the dipole approach, this is given by (5) replacing $p$ by the nucleus $A$. Qualitatively, one can roughly approximate the wavefunction as peaked at the dipole sizes around $\boldsymbol{r} \sim 1 / m_{V}=1 /\left(2 m_{f}\right)$ in the photoproduction case. Namely, $\Psi^{\gamma} \Psi^{V *} \propto \delta\left(r-1 / m_{V}\right) \delta(z-1 / 2)$ and its normalization can be obtained from the electronic decay width constraint. This is supported by recent phenomenological studies on meson production within the dipole models $[20$, 26,29]. Therefore, under this assumption the integration on longitudinal fraction $z$ and dipole size $r$ in (5) can be carried out. This procedure gives the following, up to a normalization factor:

$$
\left.\frac{\mathrm{d} \sigma_{\gamma A \rightarrow V A}^{\text {dipole }}}{\mathrm{d} t}\right|_{t=0} \propto \frac{\left[\sigma_{\text {dip }}^{\text {nucleus }}\left(s, \boldsymbol{r}^{2}=1 / m_{V}^{2} ; A\right)\right]^{2}}{16 \pi}
$$

for the nuclear photoproduction of vector mesons. The equivalent expression in the VDM approach is given by (see (13) in [7])

$$
\left.\frac{\mathrm{d} \sigma_{\gamma A \rightarrow V A}^{\mathrm{VDM}}}{\mathrm{d} t}\right|_{t=0} \propto \frac{\left[\sigma_{\mathrm{tot}}(V A)\right]^{2}}{16 \pi},
$$

where the meson-nucleus total cross section has been labeled as $\sigma_{\text {tot }}(V A)$. In [7] the following expression has been assumed for this cross section (see (12) in [7]):

$$
\sigma_{\text {tot }}(V A)=\int \mathrm{d}^{2} b\left\{1-\exp \left[-\sigma_{\text {tot }}(V p) T_{A}(b)\right]\right\},
$$

which drives the behavior on energy and atomic number of the photonuclear cross section. As discussed in detail in [8] the above expression implies that in the black disk limit the total cross section becomes equal to $\pi R_{A}^{2}$, which is the prediction of classical mechanics. In contrast, a quantum mechanical approach implies that in that limit $\sigma_{\text {tot }}=2 \pi R_{A}^{2}$. In [8] the coherent $\rho$ production in UPC was studied using the generalized vector dominance model and the quantum mechanical Gribov-Glauber approach, which implies that $\sigma_{\text {tot }}(V A)$ is given in the large coherence length limit by

$$
\sigma_{\text {tot }}(V A)=2 \int \mathrm{d}^{2} b\left\{1-\exp \left[-\frac{1}{2} \sigma_{\text {tot }}(V p) T_{A}(b)\right]\right\}
$$

On the other hand, in the QCD color dipole picture we have a model that depends basically on the different expressions for the nuclear scattering. For the first one it reads, under the assumption adopted above,

$$
\begin{aligned}
& \sigma_{\text {dip }}^{\text {nucleus }}\left(x, r \simeq \frac{1}{m_{V}}\right) \\
& =2 \int \mathrm{d}^{2} b\left\{1-\exp \left[-\frac{1}{2} \sigma_{\text {dip }}^{\text {proton }}\left(x, r \simeq \frac{1}{m_{V}}\right) T_{A}(b)\right]\right\}
\end{aligned}
$$

where the dipole-proton cross section is given by (12) for the saturation model. In particular, $\sigma_{\text {dip }}^{\text {proton }}=\sigma_{0} \simeq 23-$ $26 \mathrm{mb}$ in the case $Q_{\mathrm{sat}}^{2}(x) \gtrsim m_{V}^{2}$, while in the case where $Q_{\text {sat }}^{2}(x) \ll m_{V}^{2}$ it presents the color transparency behavior $\sigma_{\text {dip }} \simeq Q_{\text {sat }}^{2} / 4 m_{V}^{2}$. In what follows, we present a theoretical and numerical comparison among the approaches.

The dipole cross section is somewhat similar to the meson-hadron cross section, since it gives the probability of scattering of a color dipole (a $q \bar{q}$ pair) off a hadron. Therefore, we can consider them at a level of similarity. Hence, the main difference between (17) and (19) comes from the rescattering procedure. In the dipole approach, one has the standard Glauber-Gribov formalism, whereas in the VDM approach used in [7] a classical mechanical version has been used. In the color transparency regime, characteristic at intermediate energies (or light nuclei) and/or heavy mesons, both the color dipole and the meson-hadron cross section are small. This implies that the first terms in the rescatterings are the leading ones 


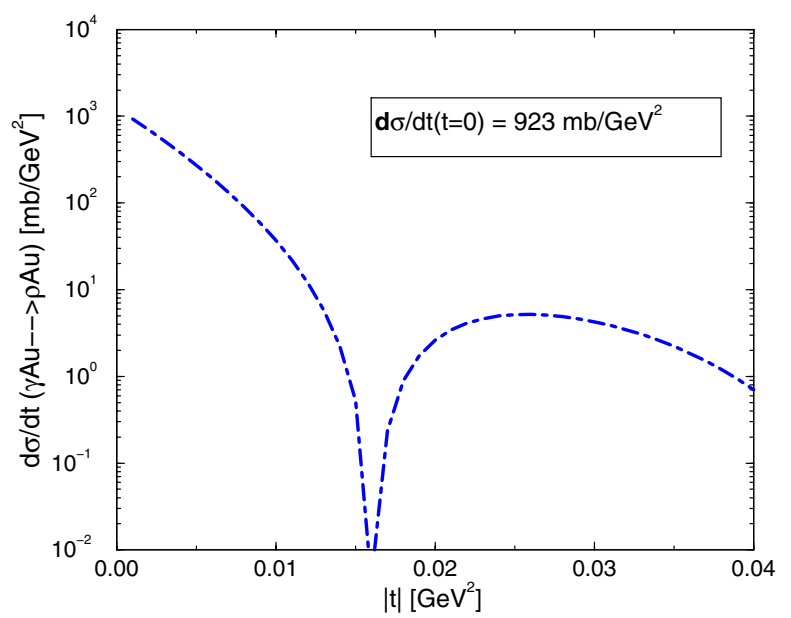

a

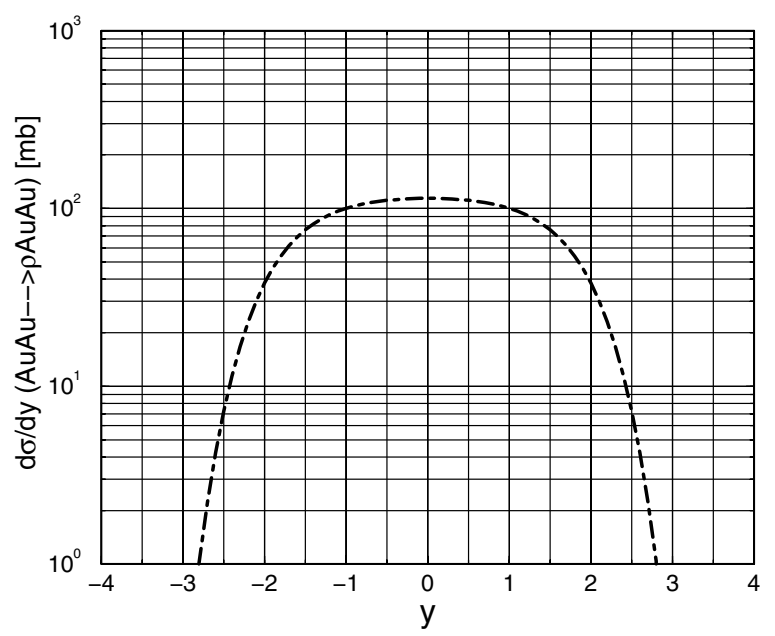

b

Fig. 3. a Momentum transfer dependence of the $\rho$ meson photonuclear production at $\mathrm{RHIC} ; \mathbf{b}$ rapidity distribution of $\mathrm{co}-$ herent $\rho$ meson production in gold-gold collisions at RHIC $\left(\sqrt{s_{N N}}=130 \mathrm{GeV}\right)$ for $\mathrm{QCD}$ dipole picture and saturation model

and (17) and (19) give quite similar results. On the other hand, at very high energies (or heavy nuclei) and/or light mesons the black disk limit is reached. Thus, one obtains for the dipole approach $\sigma_{\mathrm{dip}}^{\text {nuc }}=2 \pi R_{A}^{2}$ and for the vector meson dominance model $\sigma_{\text {tot }}(V p)=\pi R_{A}^{2}$. In this limit case the ratio $\sigma_{\gamma A}^{\text {dipole }} / \sigma_{\gamma A}^{\mathrm{VDM}}$ reaches a factor 4 . Similar arguments have been claimed in [8].

Now we compare our results with the ones in [8], where the main focus is on the $\rho$ and $J / \Psi$ production. For $\rho$ the predictions are computed only for RHIC energy $\sqrt{s_{N N}}=130 \mathrm{GeV}$ and we will consider it later on. We can anticipate that their results are closer to ours since a Glauber-Gribov approach is used in describing the scattering on nuclei. For $J / \Psi$ the theoretical approach for the photonuclear production was the collinear QCD double logarithmic approximation, where the $\gamma A \rightarrow J / \Psi A$ cross section is directly proportional to the squared nuclear gluon density distribution [45]. There, it was considered an impulse approximation (no nuclear shadowing) and a leading twist shadowing version. The impulse approximation gives a larger cross section at central rapidity (about a factor 4 higher for $\mathrm{Ca}$ and a factor 6 for $\mathrm{Pb}$ ), while at fragmentation region both approximations match each other at LHC energy for $\mathrm{Ca}$ and $\mathrm{Pb}$ nuclei. Our results are closer to their impulse approximation, which suggests nuclear shadowing could be weak for $J / \Psi$ production. This feature can be easily tested in the first experimental measurements of coherent $J / \Psi$ production on UPC's at LHC. Concerning the integrated cross section, they found $0.6 \mathrm{mb}$ for $\mathrm{Ca}$ nucleus and $70 \mathrm{mb}$ for $\mathrm{Pb}$ at LHC. Our results are $0.44 \mathrm{mb}$ and $41.5 \mathrm{mb}$, respectively. Thus, our results are about $15 \%$ lower for $\mathrm{Ca}$ and also $40 \%$ lower for $\mathrm{Pb}$. The difference between the predictions comes mostly from the distinct QCD approaches considered used and the different photon flux in the UPC calculation.

Recently, the STAR Collaboration published the first experimental measurement on the cross section of the coherent $\rho$ production in gold-gold ultraperipheral collisions at $\sqrt{s}=130 \mathrm{GeV}$ [27], providing the first opportunity to test the distinct approaches describing nuclear vector meson photoproduction. In what follows we compare our results with these experimental data and confront them with other theoretical predictions currently available. In Fig. 3a we present the momentum transfer behavior for the photonuclear $\rho$ production, which is the input for the UPC calculation. It is obtained by unfolding the integration over $|t|$ in (11). The dependence is proportional to the squared of the nuclear form factor in (10). The STAR Collaboration performed an exponential fit $\mathrm{d} \sigma_{\gamma A \rightarrow \rho A} / \mathrm{d} t \propto \mathrm{e}^{-B_{\text {nuc }}|t|}$ for this reaction, with an approximate gold radius of $R_{A u}=$ $\sqrt{4 B_{\mathrm{nuc}}}=7.5 \pm 2 \mathrm{fm}$, and obtained a forward cross section $\mathrm{d} \sigma_{\gamma A}(t=0) / \mathrm{d} t=965 \pm 140 \pm 230 \mathrm{mb} / \mathrm{GeV}^{2}$. Accordingly, we have found the value $\mathrm{d} \sigma_{\gamma A}^{\text {sat }}(t=0) / \mathrm{d} t=923 \mathrm{mb} / \mathrm{GeV}^{2}$, which is consistent with the experimental results. The usual dip occurs at $|t| \simeq 0.015 \mathrm{GeV}$, which was also found in the calculation of [8]. The rapidity distribution of the coherent $\rho$ production on UPC's is shown in Fig. 3b. The plateau at mid-rapidity remains, in contrast with the double-peak structure appearing in the calculations of [8]. However, the values at central rapidities are similar and of order $\mathrm{d} \sigma(y \approx 0) / \mathrm{d} y \simeq 100 \mathrm{mb}$.

The energy dependence of the cross section for the photoproduction of the meson $\rho$ in gold-gold ultraperipheral collisions is presented in Fig. 4. For comparison, the experimental data from the STAR Collaboration at RHIC at $\sqrt{s_{N N}}=130 \mathrm{GeV}$ [27] is also shown. Our theoretical estimations in the curves take into account the experimental cuts. The cut on the momentum transfer $|t|<0.02 \mathrm{GeV}^{2}$ slightly reduces the cross section by a few percents. In the range of rapidities $|y| \leq 3$ (left plot), at energy $\sqrt{s_{N N}}=130 \mathrm{GeV}$, we have found $\sigma_{\text {sat }}(-3 \leq y \leq 3)=410 \mathrm{mb}$ in good agreement with the STAR measurement $\sigma_{\mathrm{STAR}}(-3 \leq y \leq 3)=370 \pm$ $170 \pm 80 \mathrm{mb}$. For the cut $|y| \leq 1$ (left plot), we have obtained $\sigma_{\text {sat }}(-1 \leq y \leq 1)=221 \mathrm{mb}$, whereas the STAR result is $\sigma_{\mathrm{STAR}}(-1 \leq y \leq 1)=140 \pm 60 \pm 30 \mathrm{mb}$. In this case, our result is about $35 \%$ higher than the cen- 

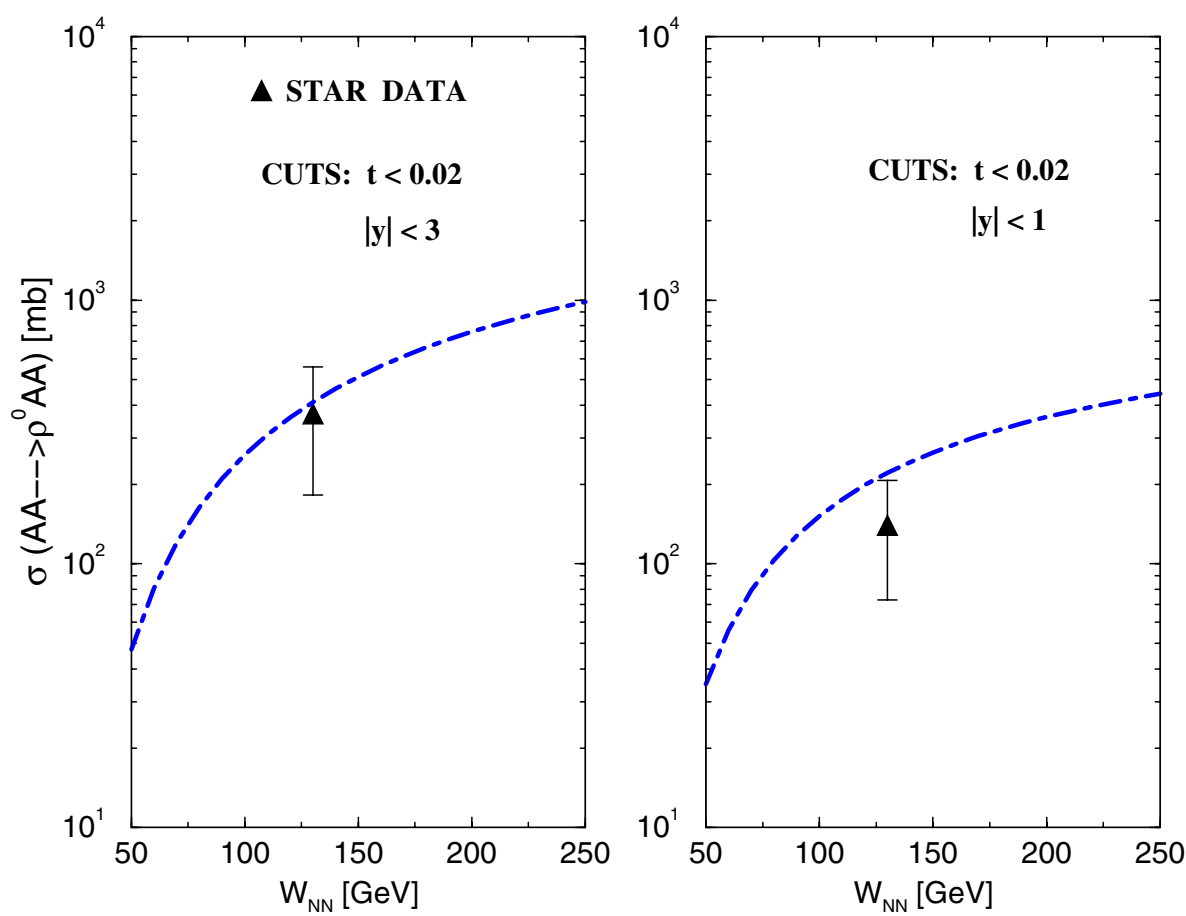

Fig. 4. Energy dependence of coherent $\rho$ meson production in gold-gold in UPC's at RHIC $\left(\sqrt{s_{N N}}=130 \mathrm{GeV}\right)$ in the QCD dipole picture and saturation model. Experimental data are from the STAR Collaboration [27] tral value of the STAR measurement. The values presented here are somewhat similar to the ones obtained in [8], which uses the generalized vector dominance model (GVDM) and the Glauber-Gribov approach, including in addition the finite coherence length effects. As discussed before, our calculation on the photonuclear cross section $\gamma A \rightarrow V A$ considers a large coherence length. This is not the case at low photon energies released at RHIC and the finite length effect could further suppress the cross section. We believe this suppression should not be strong, once they obtained $\sigma_{\mathrm{GVDM}}(-3 \leq y \leq 3)=490 \mathrm{mb}$ and $\sigma_{\mathrm{GVDM}}(-1 \leq y \leq 1)=170 \mathrm{mb}$, respectively.

Finally, let us now consider the photoproduction of vector mesons in proton-proton collisions for RHIC $(\sqrt{s}=$ $500 \mathrm{GeV})$, Tevatron $(\sqrt{s}=1960 \mathrm{GeV})$ and LHC $(\sqrt{s}=$ $14000 \mathrm{GeV}$ ) energies (for a similar analysis of the photoproduction of heavy quarks in $p p$ collisions, see [46]). In Figs. 5 and 6 , we present our predictions for the rapidity distributions and in Table 2 our results for the integrated cross section. The distribution on the rapidity has similar features as for the nuclear case, presenting a plateau at mid-rapidity and without double-peak structure. Concerning the integrated cross sections, for $J / \Psi$ production a comparison with the results obtained in [4] is possible. It is important to emphasize that in that reference a parameterization for the total $\gamma p \rightarrow J / \Psi p$ cross section is used as input in the calculations. In this case we have that our predictions are similar for RHIC energies, being approximately $10 \%$ larger for Tevatron and LHC energies, which is expected since the saturation model describes reasonably well the HERA data. Very recently, predictions for the $\rho$ and $\phi$ photoproduction in proton-proton collisions have been presented in [39]. We have that our results for $\phi$ production are $20 \%$ smaller for RHIC energies but similar
Table 1. The integrated cross section for nuclear vector mesons photoproduction at UPC's at RHIC and LHC energies

\begin{tabular}{llllll}
\hline & Heavy ion & $J / \Psi(3097)$ & $\phi(1019)$ & $\omega(782)$ & $\rho(770)$ \\
\hline RHIC & $\mathrm{SiSi}$ & $3.42 \mu \mathrm{b}$ & $612 \mu \mathrm{b}$ & $764 \mu \mathrm{b}$ & $6.74 \mathrm{mb}$ \\
& $\mathrm{AuAu}$ & $476 \mu \mathrm{b}$ & $79 \mathrm{mb}$ & $100 \mathrm{mb}$ & $876 \mathrm{mb}$ \\
$\mathrm{LHC}$ & $\mathrm{CaCa}$ & $436 \mu \mathrm{b}$ & $12 \mathrm{mb}$ & $14 \mathrm{mb}$ & $128 \mathrm{mb}$ \\
& $\mathrm{PbPb}$ & $41.5 \mathrm{mb}$ & $998 \mathrm{mb}$ & $1131 \mathrm{mb}$ & $10069 \mathrm{mb}$ \\
\hline
\end{tabular}

for LHC energy. In contrast, we have that our results for $\rho$ production reach a factor 2 smaller than the predictions from [39] for all energies. This difference is due to the sizeable importance of the threshold factor. If this factor is not taken into account, the behavior of the cross section near threshold $\mu_{\text {thres }}=\left(m_{p}+m_{V}\right)$ is overestimated. This gives an additional contribution in an integrated cross section, mostly in the $\rho$ case where the threshold scale stays at low energies $\mu_{\text {thres }}^{\rho} \approx 1.6 \mathrm{GeV}$. Namely, for light mesons, the differential cross section $\mathrm{d} \sigma / \mathrm{d} y$ receives a larger contribution from the low photon energies region which it is quite sensitive to the threshold corrections.

Finally, let us present a short discuss on the experimental feasibility of the reactions considered here. Although the vector meson photoproduction at $A A$ or $p p$ collisions is a small fraction in comparison to the total hadronic cross sections, the experimental separation of these reaction channels is possible. The rates are large even after taking into account the respective leptonic branching rations and/or acceptance estimates. The results presented in Tables 1 and 2 show that the coherent photoproduction of light mesons are very high at RHIC and LHC. For instance, the exclusive $\rho$ production coming from these 
RHIC: $\mathbf{p p} \rightarrow \mathbf{p p}+\left(\mathbf{V}_{\mathbf{M}}=\rho, \omega, \phi, J / \Psi\right)$

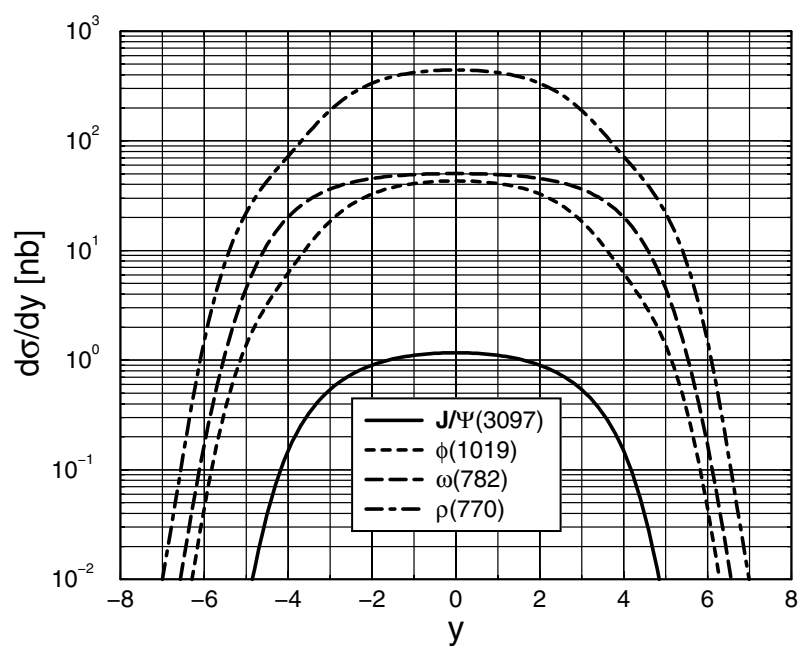

Tevatron: $\mathbf{p p}-\rightarrow \mathbf{p p}+\left(\mathrm{V}_{\mathrm{M}}=\rho, \omega, \phi, \mathrm{J} / \Psi\right)$

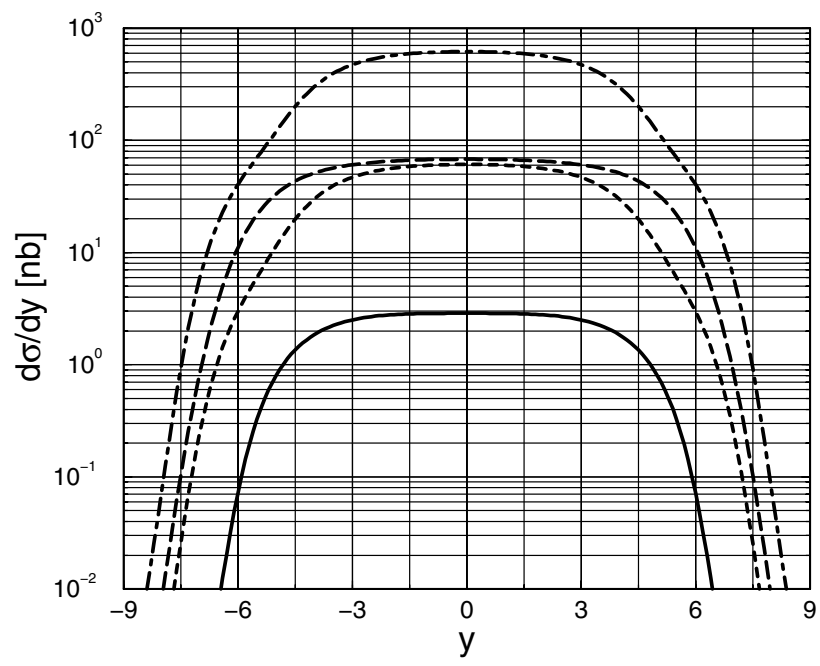

Fig. 5. The rapidity distribution for vector meson photoproduction on $p p$ or $p \bar{p}$ reactions at RHIC $\left(\sqrt{S_{N N}}=0.5 \mathrm{TeV}\right)$ and Tevatron $\left(\sqrt{S_{N N}}=1.96 \mathrm{TeV}\right)$ energies

reactions reaches $10 \%$ of the total nucleus-nucleus cross section at RHIC, whereas corresponds to $50 \%$ of the $\mathrm{PbPb}$ total cross section at LHC. As photoproduction is an exclusive reaction, $N+N \rightarrow N+N+V(N=p, A)$, the separation of the signal from hadronic background would be relatively clear. Namely, the characteristic features in photoproduction at UPC's are low $p_{\mathrm{T}}$ meson spectra and a double rapidity gap pattern. Moreover, the detection (Roman pots) of the scattered nuclei (or protons) can be an additional useful feature. In hadroproduction, the spectra on the transverse momentum of mesons are often peaked around the meson mass, $p_{\mathrm{T}} \approx m_{V}$. A experimental cut $p_{\mathrm{T}}<1 \mathrm{GeV}$ would eliminate most of the hadronic background. Hence, the rapidity cut would enter as an auxiliary separation mechanism. This procedure is specially powerful, since there will be rapidity gaps on both sides
LHC: $\mathbf{p p ~} \rightarrow \mathbf{p p}+\left(\mathrm{V}_{\mathrm{M}}=\rho, \omega, \phi, \mathrm{J} / \Psi\right)$

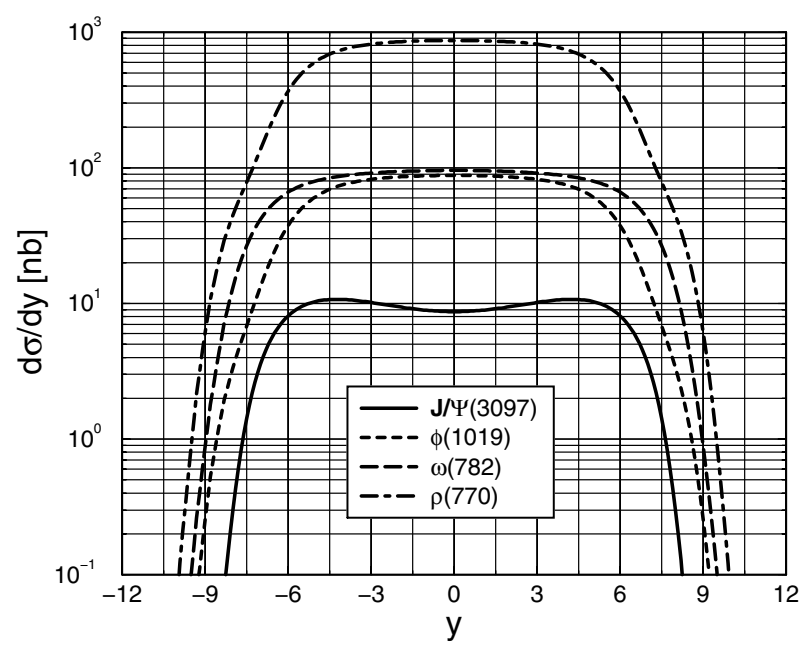

Fig. 6. The rapidity distribution for vector meson photoproduction on $p p$ reactions at LHC energy $\sqrt{S_{N N}}=14 \mathrm{TeV}$

Table 2. The integrated cross section for the photoproduction of vector mesons in $p+p(\bar{p})$ collisions at RHIC, Tevatron and LHC

\begin{tabular}{lllll}
\hline$V_{M}\left(m_{V}\right) /$ Collider & $J / \Psi(3097)$ & $\phi(1019)$ & $\omega(782)$ & $\rho(770)$ \\
\hline RHIC & $6.57 \mathrm{nb}$ & $242.4 \mathrm{nb}$ & $361.7 \mathrm{nb}$ & $2.52 \mu \mathrm{b}$ \\
Tevatron & $24.64 \mathrm{nb}$ & $476 \mathrm{nb}$ & $646 \mathrm{nb}$ & $4.84 \mu \mathrm{b}$ \\
LHC & $132 \mathrm{nb}$ & $980 \mathrm{nb}$ & $1.24 \mu \mathrm{b}$ & $9.75 \mu \mathrm{b}$ \\
\hline
\end{tabular}

of the produced meson. For numerical estimates on these cuts procedures, we quote $[11,39]$.

\section{Summary}

In summary, we have calculated the rapidity distribution and integrated cross sections of exclusive photonuclear production of vector mesons in ultraperipheral heavy-ion collisions within the QCD color dipole picture, with particular emphasis on the saturation model. This is motivated by the good agreement of this model in systematically describing the current data on vector meson photoproduction in scattering on protons and its reliable estimates for scattering on nuclei. The cross sections for the $A+A \rightarrow A+A+V(V=\rho, \omega, \phi, J / \Psi)$ process were computed and theoretical estimates for scattering on both light and heavy nuclei are given for RHIC and LHC energies. The rates are very high, mostly for light mesons and at LHC energies. In particular, we compare our prediction for the coherent $\rho$ meson production with RHIC data at $\sqrt{s_{N N}}=130 \mathrm{GeV}$. The corresponding results are in good agreement with the experimental results when considered the cuts on momentum transfer and on rapidity. We also have contrasted our results with the current models which consider vector dominance (VDM) or generalized vector meson dominance (GVDM) and Glauber-Gribov formalism for the nuclear scattering. We have pointed out the 
main sources of the discrepancies among those models and our estimates. In addition, the cross sections for the $p+p(\bar{p}) \rightarrow p+p(\bar{p}) V$ process are computed and theoretical estimates are given for the hadronic colliders RHIC and LHC in their $p p$ mode and Tevatron. Finally, the experimental feasibility and signal separation on the reaction channels presented here are briefly discussed. Although the rates are lower than hadroproduction, the coherent photoproduction signal would be clearly separated by applying a transverse momentum cut $p_{\mathrm{T}}<1$ and two rapidity gaps in the final state.

Acknowledgements. One of us (M.V.T.M.) thanks the support of the High Energy Physics Phenomenology Group, GFPAE IF-UFRGS, Brazil. This work was partially financed by the Brazilian funding agencies CNPq and FAPERGS.

\section{References}

1. C.A. Bertulani, G. Baur, Phys. Rep. 163, 299 (1988); G. Baur, K. Hencken, D. Trautmann, S. Sadovsky, Y. Kharlov, Phys. Rep. 364, 359 (2002)

2. Ch. Hofmann, G. Soff, A. Schafer, W. Greiner, Phys. Lett. B 262, 210 (1991); N. Baron, G. Baur, Phys. Rev. C 48, 1999 (1993); M. Greiner, M. Vidovic, Ch. Hofman, A. Schafer, G. Soff, Phys. Rev. C 51, 911 (1995); F. Krauss, M. Greiner, G. Soff, Prog. Part. Nucl. Phys. 39, 503 (1997)

3. V.P. Goncalves, C.A. Bertulani, Phys. Rev. C 65, 054905 (2002)

4. S.R. Klein, J. Nystrand, R. Vogt, Phys. Rev. C 66, 044906 (2002)

5. V.P. Goncalves, M.V.T. Machado, Eur. Phys. J. C 29, 37 (2003)

6. V.P. Goncalves, M.V.T. Machado, Eur. Phys. J. C 31, 371 (2003)

7. S.R. Klein, J. Nystrand, Phys. Rev. C 60, 014903 (1999)

8. L. Frankfurt, M. Strikman, M. Zhalov, Phys. Lett. B 540, 220 (2002); B 537, 51 (2002); Phys. Rev. C 67, 034901 (2003)

9. V.P. Goncalves, M.V.T. Machado, Eur. Phys. J. C 28, 71 (2003)

10. V.P. Goncalves, M.V.T. Machado, Eur. Phys. J. C 29, 271 (2003)

11. S.R. Klein, J. Nystrand, Phys. Rev. Lett. 92, 142003 (2004)

12. L.N. Lipatov, Sov. J. Nucl. Phys. 23, 338 (1976); E.A. Kuraev, L.N. Lipatov, V.S. Fadin, JETP 45, 1999 (1977); I. I. Balitskii, L.N. Lipatov, Sov. J. Nucl. Phys. 28, 822 (1978)

13. L.V. Gribov, E.M. Levin, M.G. Ryskin, Phys. Rept. 100, 1 (1983)

14. K. Golec-Biernat, M. Wüsthoff, Phys. Rev. D 59, 014017 (1999); D 60, 114023 (1999)

15. J. Bartels, K. Golec-Biernat, H. Kowalski, Phys. Rev. D 66, 014001 (2002)

16. H. Kowalski, D. Teaney, Phys. Rev. D 68, 114005 (2003)
17. L. Favart, M.V.T. Machado, Eur. Phys. J. C 29, 365 (2003); C 34, 429 (2004)

18. A.C. Caldwell, M.S. Soares, Nucl. Phys. A 696, 125 (2001)

19. S. Munier, A.M. Stasto, A.H. Mueller, Nucl. Phys. B 603, 427 (2001)

20. J.R. Forshaw, R. Sandapen, G. Shaw, Phys. Rev. D 69, 094013 (2004)

21. V.P. Goncalves, M.V.T. Machado, Phys. Rev. Lett. 91, 202002 (2003)

22. V.P. Goncalves, M.V.T. Machado, Eur. Phys. J. C 37, 299 (2004)

23. N. Timneanu, J. Kwiecinski, L. Motyka, Eur. Phys. J. C 23, 513 (2002)

24. E. Iancu, R. Venugopalan, hep-ph/0303204

25. V.P. Goncalves, Braz. J. Phys. 34, 1406 (2004)

26. V.P. Goncalves, M.V.T. Machado, Eur. Phys. J. C 38, 319 (2004)

27. C. Adler et al. [STAR Collaboration], Phys. Rev. Lett. 89, 272302 (2002)

28. R. Nisius, Phys. Rep. 332, 165 (2000)

29. N.N. Nikolaev, Comments Nucl. Part. Phys. 21, 41 (1992); O. Benhar, B.Z. Kopeliovich, C. Mariotti, N.N. Nikolaev, B.G. Zakharov, Phys. Rev. Lett. 69, 1156 (1992); O. Benhar, S. Fantoni, N.N. Nikolaev, B.G. Zakharov, J. Exp. Theor. Phys. 84, 421 (1997) [Zh. Eksp. Teor. Fiz. 111, 769 (1997)]; N.N. Nikolaev, J. Speth, B.G. Zakharov, Phys. Atom. Nucl. 63, 1463 (2000) [Yad. Fiz. 63, 1463 (2000)]; I.P. Ivanov, N.N. Nikolaev, JETP Lett 69, 294 (1999); Acta Phys. Polon. B 33, 3517 (2002); I.P. Ivanov, Phys. Rev. D 68, 032001 (2003)

30. N.N. Nikolaev, B.G. Zakharov, Phys. Lett. B 332, 184 (1994); Z. Phys. C 64, 631 (1994)

31. E. Gotsman, E. Levin, U. Maor, Nucl. Phys. B 464, 251 (1996)

32. V. Barone, E. Predazzi, High-energy particle diffraction (Springer-Verlag, Berlin Heidelberg 2002)

33. J.A. Crittenden, Exclusive production of neutral vector mesons at the electron proton collider HERA (SpringerVerlag, Berlin Heidelberg 1997) [hep-ex/9704009]

34. I.P. Ivanov, N.N. Nikolaev, A.A. Savin, hep-ph/0501034

35. N. Armesto, Eur. Phys. J. C 26, 35 (2002)

36. V.P. Goncalves, M.V.T. Machado, Eur. Phys. J. C 30 , 387 (2003)

37. M. Drees, D. Zeppenfeld, Phys. Rev. D 39, 2536 (1989)

38. B.A. Kniehl, Phys. Lett. B 254, 267 (1991)

39. J. Nystrand, Nucl. Phys. A (to appear), hep-ph/0412096

40. H.G. Dosch, T. Gousset, G. Kulzinger, H.J. Pirner, Phys. Rev. D 55, 2602 (1997)

41. A.G. Shuvaev, K.J. Golec-Biernat, A.D. Martin, M.G. Ryskin, Phys. Rev. D 60, 014015 (1999)

42. C. Brenner Mariotto, M.B. Gay Ducati, M.V.T. Machado, Phys. Rev. D 66, 114013 (2002)

43. V.N. Gribov, Sov. Phys. JETP 29, 483 (1969); 30, 709 (1970)

44. C.W. De Jager, H. De Vries, C. De Vries, Atom. Data Nucl. Data Tabl. 14, 479 (1974)

45. S.J. Brodsky, L. Frankfurt, J.F. Gunion, A.H. Mueller, M. Strikman, Phys. Rev. D 50, 3134 (1994)

46. V.P. Goncalves, M.V.T. Machado, Phys. Rev. D 71, 014025 (2005) 